\title{
"It's like a mirror image of my illness": Exploring Patient Perceptions About Illness Using Health Mind Mapping-a Qualitative Study
}

\author{
Pablo Buitron de la Vega, M.D., M.S.c., ${ }^{1,2}$, Christopher Coe, M.D. ${ }^{2}$, \\ Michael K. Paasche-Orlow, M.D., M.A., M.P.H. ${ }^{1,2}$, Jack A. Clark, Ph.D. ${ }^{3}$, Katherine Waite, B.A. ${ }^{1}$, \\ Maria Jose Sanchez, M.D. ${ }^{4}$, Emily Armstrong, B.A. ${ }^{7}$, and Barbara G. Bokhour, Ph.D. ${ }^{3,5}$ \\ ${ }^{1}$ General Internal Medicine, Boston Medical Center, Boston, MA, USA; ${ }^{2}$ Boston University School of Medicine, Boston, MA, USA; ${ }^{3}$ Boston University \\ School of Public Health, Boston, MA, USA; ${ }^{4}$ Cambridge Health Alliance, Cambridge, MA, USA; ${ }^{5}$ Center for Healthcare Organizations and \\ Implementation Research, Edith Nourse Rogers VA Medical Center, Bedford, MA, USA.
}

BACKGROUND: A patient's self-management of chronic disease is influenced in part by their explanatory model of illness (EMI) and daily lived experiences (DLE). Unfortunately, assessing patient's EMI and using this information to engage patients in chronic illness self-management continues to be a challenge.

OBJECTIVE: "Health mind mapping" (HMM) is a novel process that captures a patient's EMI and DLE through the use of a graphic representation of ideas. We aimed to explore patient's experiences using HMM.

DESIGN: Qualitative study utilizing semi-structured interviews.

PARTICIPANTS: Adult patients with uncontrolled type 2 diabetes from a primary care clinic.

APPROACH: A facilitator guided 20 participants through the process of developing a health mind map. Subsequently, each participant completed a semi-structured interview about their experience with the process and perceptions about how their maps could be used. The process and interviews were video and audio recorded. We conducted a content analysis of the maps and a thematic analysis, using an inductive approach, of the interview data.

RESULTS: Participants explored a wide range of EMs and DLEs in their HMM process. Participants reported that the HMM process (1) helped to develop insight about self and illness; (2) was a catalyst for wanting to take actions to improve illness; and (3) represented an opportunity to actively share illness experiences. They reported potential uses of the map: (1) to communicate about their illness to others in their social network; (2) to communicate about their illness to providers; (3) to help others with diabetes manage their illness; and (4) to encourage ongoing engagement in diabetes self-care.

CONCLUSIONS: Participants reported that HMM helped them develop new insight about their illness and was a catalyst for encouraging them to take control of their

Electronic supplementary material The online version of this article (https://doi.org/10.1007/s11606-018-4557-9) contains supplementary material, which is available to authorized users.

Received July 11, 2017

Revised February 6, 2018

Accepted June 18, 2018

Published online July 10, 2018 illness. HMM has the potential to facilitate communication with providers and engage patients in collaborative goal setting to improve self-care in chronic illness.

KEY WORDS: health mind mapping; explanatory models of illness; daily lived experiences; diabetes.

J Gen Intern Med 33(10): 1692-9

DOI: $10.1007 / \mathrm{s} 11606-018-4557-9$

(c) Society of General Internal Medicine 2018

\section{INTRODUCTION}

Patients with chronic illnesses like diabetes face significant challenges with self-management. ${ }^{1}$ Self-management for patients with diabetes mellitus include monitoring daily glucose levels, regulating medications, exercise, and diet in the context of their work, home, and leisure lives. ${ }^{2}$ These activities represent a significant challenge for many patients, especially those with limited social support, time constraints, and limited health literacy. ${ }^{3,4}$ Different approaches have been proposed to improve self-management, however they have been shown to have minimal clinical impact and to not be sustained by patients in the long term. ${ }^{5-9}$

Two key factors associated with successful selfmanagement are patient's explanatory models of illness (EMI) and patients' daily lived experiences (DLE). ${ }^{10}$ EMIs refer to how people understand the causes and mechanisms of an illness, as well as how they perceive the course of their illness, their symptoms, and effects of treatment. ${ }^{10,11} \mathrm{DLE}$ refer to the patient's social context, routines, habits, and competing health problems. ${ }^{10}$ Providers who understand their patient's EMI and DLE could be more effective at personalizing treatments, educating patients using language that is salient and that patients can understand, and validating patient's emotional states. $^{12,13}$ Different methods have been proposed to elicit EMIs and DLEs. ${ }^{10,14-17}$ Drawing is one method used to explore how patients think about their illness. ${ }^{17-21}$ Patients drawings give an opportunity to explore patient's beliefs and perceptions about their illness and the treatment they are receiving. ${ }^{17,18}$ Despite their significance, using drawings to 
assess patients' perceptions are not systemically used in clinical care. Thus, it is important to develop novel approaches that can be integrated into routine patient care so clinicians can understand and address the social and behavioral aspects of their patients' illnesses.

One novel approach is the use of health mind mapping to elicit patients' EMI and DLE. Health mind mapping (HMM) is a process developed by the principal investigator (PI) based on the concept of mind mapping - a "visual, non-linear representations of ideas and their relationships" 22 that was formally developed by Tony Buzan. ${ }^{23}$ HMM consists of exploring patients' perceptions by asking them questions using a semi-structured interview and organizing answers by drawing a "mind map." The semi-structured interview includes 14 domains derived and adapted from Kleinman's EMI questions, ${ }^{24}$ cultural formulation interview, ${ }^{25}$ and from a study that assessed explanatory models of diabetes. ${ }^{26}$ The semistructured questions are included in Appendix 1. After the interviewer asks each question, the patient uses mind mapping to organize the answers. The final product of asking the semi-structure interview questions and organizing the answers using mind mapping is called a health mind map. The structure of a health mind map has three basic elements as illustrated in Figure 1: (1) central idea (represented by the patient's body); (2) parent branches; and (3) child branches. In HMM, the focus is on patients' experiences of their health and illness. The end product is a graphic depiction of the patient's EMI and DLE that belongs to the patient and can be taken home and shared with friends, family, or care providers. The aim of the study was to examine how patients engage with and experience HMM and how this process is viewed by patients for their diabetes self-management.

\section{METHODS}

\section{Participants and Recruitment}

We recruited patients from primary care clinics at Boston Medical Center (BMC). All patients were adults ( $>18$ years old) with inadequately controlled diabetes type 2 (hemoglobin A1C > 7), spoke English or Spanish, and reported they could read and write in either language. Patients who were blind, cognitively impaired, or otherwise unable to consent

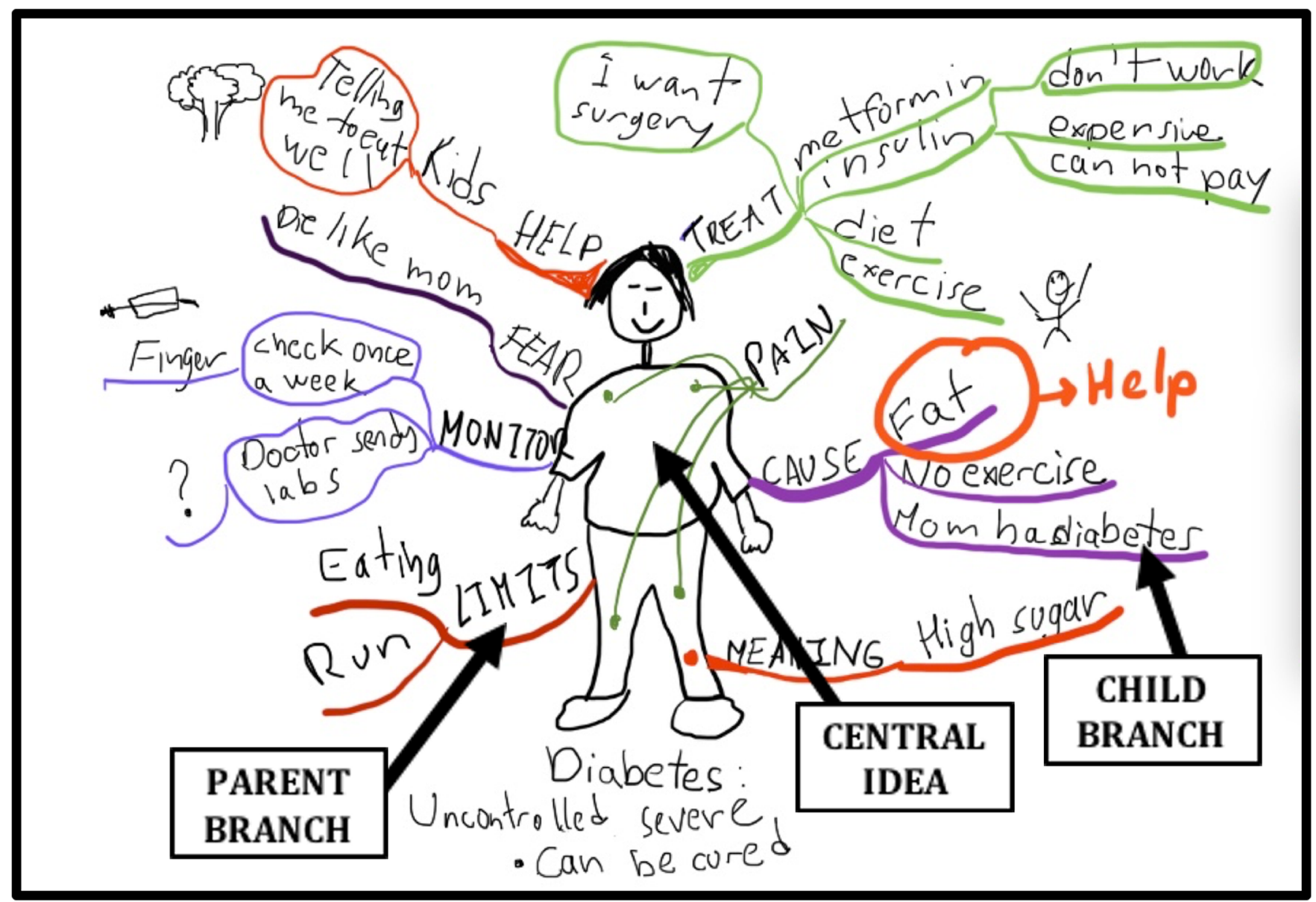

Fig. 1 Health mind map structure. The central idea represents the main topic of the mind map; parent branches represent ideas related to the central idea, and child branches represent ideas related to the parent branches. 
independently were excluded. This study was approved by the Institutional Review Board (IRB) at BMC.

Primary care providers identified patients that met criteria and obtained permission for study staff to contact potential participants and provide a brief explanation about the study. Patients who agreed to participate were provided written informed consent and completed a brief demographic questionnaire before they started HMM.

\section{Procedures}

A HMM trained facilitator met privately with each participant and guided the process. Facilitators were two medical assistants (MA), one care coordinator (CC), and one research assistant (RA). Each one of the facilitators was individually trained in a single session by the PI in how to guide research participants to develop the maps. The training time for each facilitator varied between 40 and $60 \mathrm{~min}$. In order to check for consistency, the PI checked the maps developed during the individual session to confirm that basic elements (central idea and branches) were included.

\section{HMM Facilitation Process}

The facilitator (MA, CC, or RA depending of their availability) guided the patient to develop a health mind map. The patient was first asked to draw a representation of their body (central idea) in the center of a blank sheet of paper. After drawing the central idea, the facilitator asked the patient semistructured questions (Appendix 1) to elicit their perspectives regarding their illness. While the patient verbally answered the questions, the facilitator asked the patient to write down their answers in just a few words using the mind mapping technique. The last question asked the patient to identify and circle one area in their maps that they would like to work on.

\section{Data Collection}

Qualitative Interview. After completing HMM with the assistant, participants underwent a semi-structured qualitative interview with the PI. Sample questions are provided in Table 1. The interview was recorded and transcribed verbatim and covered questions in four areas-experience of participation, perceptions of what was meaningful

Table 1 Semi-structured Sample Questions

\begin{tabular}{ll}
\hline \hline Topic & Participant interview guide \\
\hline Experience of participation & $\begin{array}{l}\text { What was it like for you to do the } \\
\text { health mind map? }\end{array}$ \\
$\begin{array}{l}\text { Perception of what was } \\
\text { meaningful about HMM }\end{array}$ & $\begin{array}{l}\text { yourself and andhing new about } \\
\text { the health mind map? }\end{array}$ \\
$\begin{array}{l}\text { Potential uses of health mind } \\
\text { maps }\end{array}$ & $\begin{array}{l}\text { Now that you have finished your map: } \\
\text { What do you think you might do with it } \\
\text { in the future }\end{array}$ \\
$\begin{array}{l}\text { Wharing the Health Mind } \\
\text { Map }\end{array}$ & $\begin{array}{l}\text { Whap with you think about sharing your } \\
\text { map with else }\end{array}$ \\
\hline
\end{tabular}

about HMM, potential uses of HMM and how they might share the maps with others (see Table 1). At the end of the interview, the maps were copied and the original was given to the participant. Participants received a \$20 certificate for their participation.

\section{Data Analysis}

We conducted a content analysis of all maps to identify what types of information and thoughts patients demonstrated in each of the domains. We reviewed HMM and synthesized words into broader categories. Qualitative interviews were transcribed verbatim. Transcriptions were uploaded to NVIVO version 11 . We conducted a thematic analysis using an inductive approach at a semantic level to identify how patients perceived and were impacted by the process. ${ }^{27}$ Two of the authors (PB, CC) independently read and coded two participant transcripts, then met to compare and discuss similarities and differences in definitions of codes. Initial code definitions were then reviewed with a third investigator (BB). Afterwards, the two (PB, CC) investigators inductively coded five interviews concurrently to develop one master code list, and then the remaining interviews were independently coded. The coders met frequently to discuss coding progress and resolve any differences. Finally, when coding was completed, all three investigators met to discuss and synthesize codes into broader themes.

\section{RESULTS}

In total, 20 participants completed the HMM process and qualitative interview. The majority were female $(65 \%)$, aged 41-74 and had mixed levels of education. Characteristics of participants are displayed in Table 2. Analysis of interviews resulted in thematic saturation after 20 interviews.

Table 2 Participant Characteristics

\begin{tabular}{ll}
\hline \hline Age & \\
Range & $41-74$ \\
Mean & 59.25 \\
Sex & 7 \\
Male & 13 \\
Female & \\
Race & 16 \\
African American & 2 \\
White & 2 \\
Hispanic & 1 \\
Educational Status* & 3 \\
Elementary school & 8 \\
Some high school & 1 \\
High school graduate & 4 \\
Some college/technical school & 1 \\
Completed college & \\
Some graduate school & $7.1-12.2$ \\
Hemoglobin A1C & 8.9 \\
Range & \\
Mean &
\end{tabular}

*Data missing for two participants 
Figure 2 is an example of a map developed by one participant. Her map shows how transportation represents a barrier to control of her illness. It is important to note that by using EMI questions as prompts, she depicted both domains of her EMI (e.g., thinking that weight, pregnancy, food are causes of her DM), and concepts that relate to her DLE (e.g., taking classes, exercising). This participant selected "WEIGHT" as the area on her map that she wanted to work on. This example is similar in content to others' maps in each domain, and the content analysis of other participants' maps is shown in Table 3.

Mostly, patients were positive about their experience. Only one participant did not want to take her map home because she felt it reminded her of negative emotions associated to her illness. Participants reported HMM was beneficial in several ways. They described an impact on their awareness about their illness, with the potential to transform their approach to managing their diabetes. Participants discussed two primary domains regarding HMM: (A) perceptions of the process itself, and (B) the potential use of health mind maps in the future.

\section{Participant Perceptions About Health Mind Mapping Process}

Three main themes emerged to illustrate how participants viewed HMM: (1) HMM helps to develop insight about self and illness; (2) HMM can be a catalyst for wanting to take actions to improve illness; and (3) It can be an opportunity to actively share illness.

1. HMM helps to develop insight about self and illness:

Participants perceived that HMM helped them formulate and exhibit perspectives and knowledge regarding their diabetes that were in their mind, yet not fully acknowledged.

I'll have something to look at to be more focused on. I mean, this is like looking in a mirror, if you ask me. It's like a mirror image, because you can't see this if you look in the mirror, but if I put this on my mirror then I could see it, and I can read it. (V10)

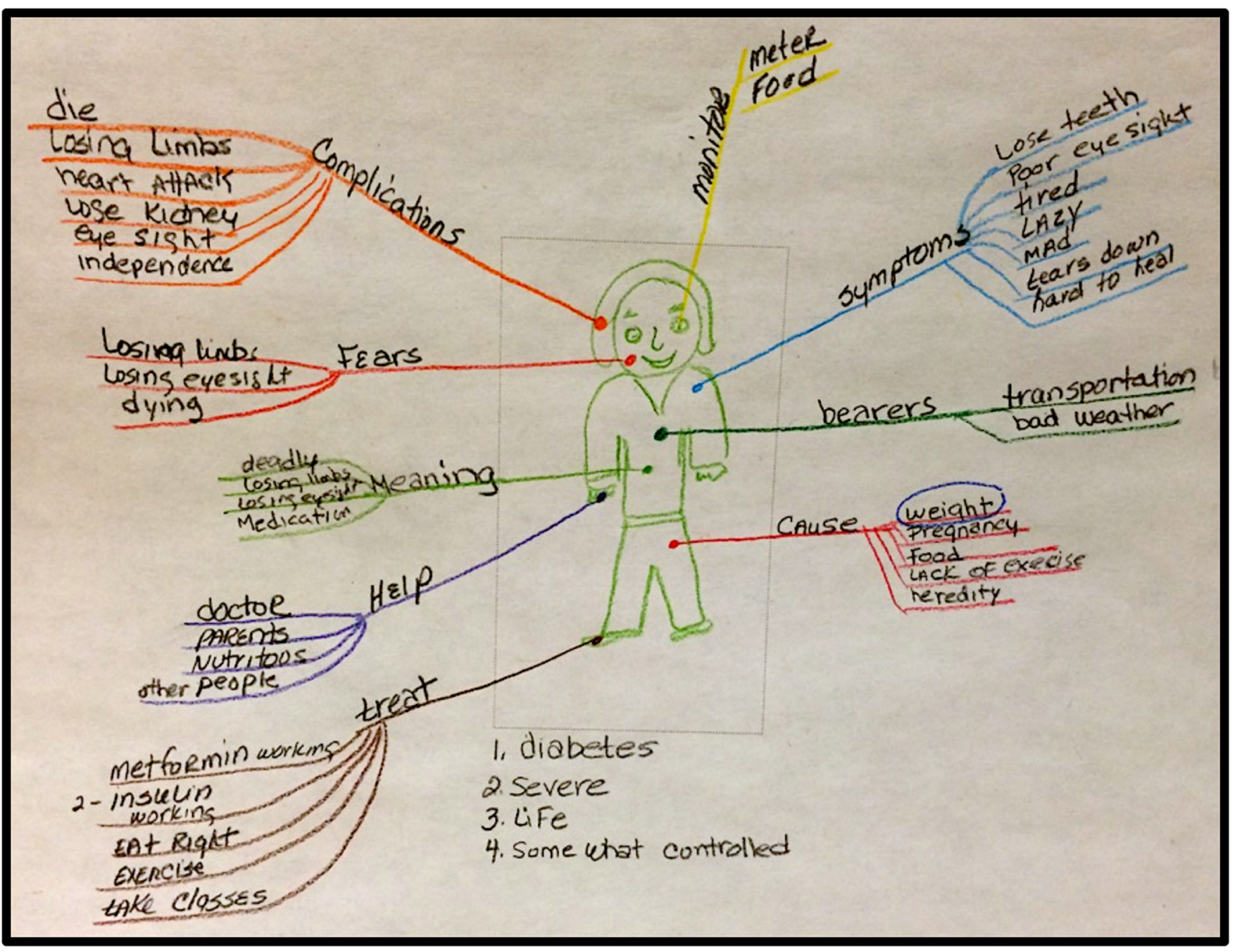

Fig. 2 Health mind map of a middle-aged female with obesity and diabetes. 
Table 3 Analysis of Health Mind Map Content

\begin{tabular}{|c|c|}
\hline $\begin{array}{l}\text { Explanatory model of } \\
\text { illness domain }\end{array}$ & Examples of participant responses \\
\hline Meaning & Threat to life*; torment/ruin family \\
\hline Perception of control & Uncontrolled* \\
\hline Cause & Poor diet*, weight gain*; lack of sleep \\
\hline Symptoms & $\begin{array}{l}\text { Feeling sad/down*, weight gain*, eye } \\
\text { problem*; thinking about diabetes all the } \\
\text { time }\end{array}$ \\
\hline Complications & $\begin{array}{l}\text { Death*, heart damage*, losing limbs*; } \\
\text { losing freedom }\end{array}$ \\
\hline Monitoring & $\begin{array}{l}\text { Checking blood sugar*, A1c*; amount of } \\
\text { water to drink }\end{array}$ \\
\hline Treatment & $\begin{array}{l}\text { Diet*, medications*, avoiding all } \\
\text { medications, they are bad for you }\end{array}$ \\
\hline Support & Doctor*, family*; books \\
\hline Barriers & $\begin{array}{l}\text { Money*, stress*; thinking about diabetes } \\
\text { all the time }\end{array}$ \\
\hline Fears & $\begin{array}{l}\text { Loss of body parts*; feeling stress for not } \\
\text { controlling diabetes }\end{array}$ \\
\hline
\end{tabular}

*Five or more participants wrote as a response in their health mind map

The participant expressed that seeing the finalized map (Fig. 2) made her aware that her knowledge about diabetes was much more extensive than what she initially thought.

Other participants expressed that besides externalizing perspectives and ideas it also helped them express feelings and fears about their illness. In response to a question about what she would tell others about the process, one woman replied:

No one has ever asked me these questions, so that enabled me to go as deep as I did, being honest and looking at it on paper. Inform them that the person might have to do some soul searching. (V2)

By talking and writing about the meaning of her illness, HMM represented an opportunity not only to discuss the different areas of her EMI, but also an opportunity to bring her feelings to the surface.

2. HMM can be a catalyst for wanting to take actions to improve illness:

Beyond gaining some insight into the meaning of their illness, participants went further to describe how the HMM process was a catalyst for them to want to engage in healthy behaviors.

It was hard because I'm seeing myself writing this stuff down and-like when I'm at home, I don't think about stuff like that. So for me to come here and sitting here, write this stuff down, now I get it, that I have to like take control of my diabetes. I can't let diabetes control me. (V9)

This participant had not reflected on these areas before. Through HMM, the participant had the opportunity to reflect by verbally answering questions and by synthesizing and writing the information on the paper using the mind mapping technique. This opportunity for reflection seemed to engage participants towards an intention to change behaviors to achieve better control of their illness.

3. Opportunity for participants to actively share their illness:

The participants described feeling more active in this process, contrasting it with other educational programs in which they had previously participated.

It's more meaningful to write it, instead of just hearing it, you know? I mean, it becomes more embeddedwise, because you can look at it. I mean, just if I'm in a classroom, and somebody is talking about it, you just sit in there. Your mind could be wandering somewhere else. Because they're speaking, you know? But this way, I had the chance to be active, because I had to put my mind to what I'm doing on paper. (V10)

Writing down ideas about her illness gave this participant a sense of being involved in a meaningful activity, and as a consequence, she felt engaged in the process, through actively talking, writing and synthesizing her perspectives on diabetes. Actively developing a visual tool seems to help participants get more involved in the process of sharing their illness compared to other interviews or group visits where they seemed to feel like passive participants.

\section{Moving Beyond the Process-Participant Reports of the Potential Use of Health Mind Maps in the Future}

When asked about what they might do with their health mind maps, four main themes emerged: (1) Communicate their illness to others in their social network; (2) Communicate about their illness to providers; (3) Share to help others with diabetes; (4) Use to encourage ongoing engagement in diabetes self-care.

- Using it to communicate their illness to others in their social network:

Almost all participants indicated that they would use their maps to share their illness with others in their social network, either to help them understand what they are going through or to elicit help from them.

My husband [whom she would like to share the HMM with]. Just so that he'd be aware of what it is being diabetic and how I feel or how I was able to map it out. That way he might understand. (V6)

These reflect how important it is to the participants to make others aware about what they are going through because of their illness. These participants indicated that having a written document that depicts their perceptions about diabetes could help them have an outline that can facilitate the sharing process with others. 
- Communicating their illness to providers:

Other participants said that using the maps would also facilitate sharing their understandings of diabetes with their providers.

They [providers] will get an understanding of where I'm at with my — with my diabetes, you know. Because I think maybe, you know, that they think I don't care, or it's just a game or something, you know. I do care. I like to - I like - I like the knowledge. (V14)

This participant and others in the study valued the importance of having the provider understand her perceptions regarding diabetes. The purpose was twofold. First, sharing would make the provider aware of the different domains that affect their diabetes. Second, sharing the HMM would demonstrate how committed she was regarding the treatment plan. Participants indicated that they could use this tool to demonstrate providers that they care, are engaged and want to better manage their illness.

- Sharing to help others with diabetes:

A number of participants mentioned they would like to use their HMM to teach others in their social networks who have diabetes about managing their illness.

My brother has diabetes and I would tell them to take a look at that and see, ask them where they might be having problems. And tell them what I do about it when I have problems, the same problems. (V13)Participants expressed enthusiasm for using their maps as a tool to facilitate sharing of their experiences and knowledge about diabetes management with others. HMM provide a platform for discussion and thus could be used as an education tool amongst diabetic peers.

- Empowering ongoing engagement in diabetes self-care: Participants expressed that having this tool at home could help them engage with their daily self-management tasks related to their illness.

I'll have it as a stepping stone, a learning tool, you know? I think - like I said, I think I'll just put it on the refrigerator, so, you know, like I will see it all the time, every time I go in the refrigerator, I will be able to look at it. (V1)Participants expressed the challenge of taking action and staying the course on their self-care activities. They saw the maps as a constant reminder of what they should and should not do to manage their diabetes and help them better engage with ongoing selfmanagement.

Participants' active engagement in responding to questions by drawing the map seemed to facilitate future reflection on their progress towards their goals for self-management. Participants also said that the act of producing the map encouraged them to continue to reflect on progress towards their goals. They further expressed that having new perspectives about their illness or just being aware of all the domains could empower them to start developing new goals to better control their illness. Additionally, participants stated that keeping the map where they could see it regularly would remind them about their diabetes and encourage further reflection, learning, and engagement in self-management.

\section{DISCUSSION}

Patients felt that Health Mind Mapping helped them gain clarity about their illness perspectives. In addition, patients viewed HMM as a process that could help them develop a sense of responsibility for their own life and illness. Using this novel approach has the potential to facilitate patient engagement in self-management, a key to improving diabetes outcomes.

HMM participants gained insight about their Diabetes by having the opportunity to describe the meaning and experiences associated with their illness. Participants described gaining increased insight into their diabetes through engaging in the HMM process. HMM uses two different cognitive activities to explore illness perceptions: drawing and writing. Prior studies using drawing techniques to capture illness perceptions have also reported that drawing helps patients make sense of what they felt by creating a space for reflection and even playfulness. ${ }^{20,21}$ Prior research on expressive writing in which participants explore one's innermost thoughts and feelings ${ }^{28}$ through written narrative showed that this activity helped patients in the process of self-understanding. ${ }^{29,} 30$ However, patients with limited education and health literacy may not be able to complete written narrative. Our study shows that HMM was viewed as a form of expressive writing and drawing that helped participants share deep emotions regarding their illness. Yet, HMM does not require a traditional narrative approach; indeed, the "mind mapping" technique has been used effectively in childhood education. ${ }^{31}$ Thus, in theory, HMM could be an important component of interventions across health literacy levels. Future studies that use HMM should aim to include broader health literacy levels in order to clearly understand if patients with lower levels of education or health literacy are able to perform this process. Other studies of EMIs questionnaires mainly concentrate on exploring the answers to these questions; our study advances the literature by also exploring patients' perceptions of the 
experience of completing such questionnaires and how they might use the information in the future.

Participants who engaged in HMM viewed the maps as a mechanism for sharing their illness experience with their providers and for helping providers understand their own commitment to self-management. HMM is novel as it offers patients an opportunity to reflect about their illness and also creates a tangible tool that can be longitudinally shared with others. Similarly, prior studies have shown that visual representations of illness helped patients to communicate information to others in a format that suited their style. ${ }^{20}$ It is well known that traditional patient-provider interactions are usually asymmetric and characterized by the provider being the main driver of the conversation. ${ }^{32}$ If the patients' willingness to share their maps translates into actually sharing their view of their illness with providers, this could create opportunities for new power dynamics. This could also translate into patients feeling more empowered to participate in shared decision making. Future studies are needed to look into the actual effects that HMM could have on patient-provider dynamics and interactions between patients and their social network.

In our study, participants expressed that HMM functioned as a catalyst for wanting to take actions to improve their illness. Prior studies have shown how expressive writing gave individuals a sense of control over their lives and improved attitudes and resilience towards their illness. ${ }^{33,}{ }^{34}$ Our participants expressed that HMM could help them accomplish and develop new goals related to their illness. A possible explanation of this potential effect is that HMM gave patients the opportunity to reflect on their illness and goals differently by developing a graphic depiction that mirrors their thought process. Having the option to take their map home could provide further opportunities to reflect and develop new goals and strategies to improve control of their illness. Future research is needed to assess if the sense of empowerment that our patients reported they achieved through HMM will persist over time. As our results are only based on participant's selfreported behavior changes (e.g., wanting to eat healthier or planning to start exercising), it will also be important to understand if this sense of empowerment will translate into changes in their behavior.

Many interventions to improve self-management require a significant amount of resources in order to be adequately implemented. It is thus notable that the HMM facilitators were non-medical personnel and were effectively trained with a 1-h session. As participants did not develop their Health Mind Maps during their regular care, further studies are needed to test if HMM is a feasible process that could be implemented in the regular care of patients with chronic diseases.

None of the participants mentioned disadvantages that could be related to the HMM process. During the analysis of the recordings and videos, we noticed that participants had some initial trouble understanding how to use mind mapping technique. By the end of the process, however, participants seemed to have acquired the skills to continue drawing their health mind maps independently.

There are several limitations to our study. The PI and developer of HMM obtained consent for the study and interviewed the participants. This could have biased the participants' answers towards reporting positive perceptions regarding HMM during the interview. Although our goal was to develop a process that could give a voice to patients across health literacy levels, we excluded participants in this study who reported that they could not read and write. Future research is needed to examine HMM for people with more limited literacy skills. It is important to mention that almost all participants were African American (AA), as this is the population served at the study clinic. Race and ethnicity was not a focus of the study; however, future work might explore differences in process and maps for different groups. Even though most of the facilitator-participant encounters were racially discordant, most of the participants mentioned feeling comfortable discussing their illness with facilitators. Another limitation was the fact that our analysis of the HMM process was mainly based on participant's perceptions. Even though participants stated that they would like to continue using HMM during their care, we did not follow up with them to ask how and why they ended up using or not using it. It is also unclear if the empowerment gained at the end of the process will persist when the participants get back to their lives. While these findings are promising, future work is needed to ascertain if HMM leads to changes in self-care and outcomes.

Exploring patients' perceptions of their illnesses and the context in which they manage their illness is critical to fostering better chronic illness self-management. Our findings suggest that HMM could potentially be a standard structured process to collect important information about the patient's EMI and DLE. Additionally, it could help patients to gain insight into their illness and empower them to follow and improve their self-management skills. HMM could act as an aid to help patients feel empowered to share information with their providers and their social network. Even further, our participants described the possibility of using health mind maps as a means to help others engage in improving their own health.

Contributors: Facilitators involved in the health mind mapping process: Katherine Waite, Jocelyn Hernandez, Eunice Barros, Yenealem Teklemarian. Briana Dukas and Lori Henault for supporting the facilitators and recruitment of patients during the project. Carol Pineiro for her work as an editor of manuscript. Rodolfo Cabrera and Tom Kavanaugh for their input during the development of Health Mind Mapping.

Prior presentations: Poster presentation at the 2016 SGIM Annual Meeting in Florida. Oral abstract presentation at the 2017 ICCH \& HARC Annual Research Conference in Maryland.

Corresponding Author: Pablo Buitron de la Vega, M.D., M.S.c.; General Internal MedicineBoston Medical Center, Boston, MA, USA (e-mail: pbuitro1@bu.edu). 


\section{Compliance with ethical standards:}

This study was approved by the Institutional Review Board (IRB) at $B M C$.

Conflict of interest: The authors declare that they have no conflict of interest.

\section{REFERENCES}

1. Zulman DM, Jenchura EC, Cohen DM, Lewis ET, Houston TK, Asch SM. How can eHealth technology address challenges related to multimorbidity? perspectives from patients with multiple chronic conditions. Journal of general internal medicine. 2015; 30:1063-1070.

2. Greenfield S, Kaplan SH, Ware JE, Jr, Yano EM, Frank HJ. Patients participation in medical care: Effects on blood sugar control and quality of life in diabetes. J Gen Intern Med. 1988; 3:448-457.

3. Nagelkerk J, Reick K, Meengs L. Perceived barriers and effective strategies to diabetes self-management. J Adv Nurs. 2006; 54:151-158.

4. Kim S, Love F, Quistberg DA, Shea JA. Association of health literacy with self-management behavior in patients with diabetes. Diabetes Care. 2004; 27:2980-2982

5. Halligan A, Cullen R, Squire S, Hill P. The expert patients programme. Clinical Governance: An International Journal. 2006; 11:17-21.

6. Vadiee M. The UK "Expert patient program" and self-care in chronic disease management: An analysis. European Geriatric Medicine. 2012; 3:201-205.

7. Wilson PM. The UK expert patients program: Lessons learned and implications for cancer survivors' self-care support programs. Journal of Cancer Survivorship. 2008; 2:45-52.

8. Jones F. Strategies to enhance chronic disease self-management: How can we apply this to stroke? Disabil Rehabil. 2006; 28:841-847.

9. May S. Self-management of chronic low back pain and osteoarthritis. Nature Reviews Rheumatology. 2010; 6:199-209.

10. Bokhour BG, Cohn ES, Cortés DE, et al. The role of patients explanatory models and daily-lived experience in hypertension selfmanagement. Journal of general internal medicine. 2012; 27:1626-1634.

11. Kleinman A. Patients and Healers in the Context of Culture: An Exploration of the Borderland between Anthropology, Medicine, and Psychiatry. Univ of California Press, 1980

12. Roter D, Hall JA. Doctors Talking with Patients/Patients Talking with Doctors: Improving Communication in Medical Visits. Greenwood Publishing Group, 2006.

13. Marvel MK, Epstein RM, Flowers K, Beckman HB. Soliciting the patient's agenda: Have we improved? JAMA. 1999; 281:283-287.

14. Lang F, Floyd MR, Beine KL, Buck P. Sequenced questioning to elicit the patient's perspective on illness: Effects on information disclosure, patient satisfaction, and time expenditure. Fam Med. 2002; 34:325-330.

15. Krupat E, Frankel R, Stein T, Irish J. The four habits coding scheme: Validation of an instrument to assess clinicians' communication behavior. Patient Educ Couns. 2006; 62:38-45.
16. Broadbent E, Petrie KJ, Main J, Weinman J. The brief illness perception questionnaire. J Psychosom Res. 2006; 60:631-637.

17. Petrie KJ, Weinman J. Patients' perceptions of their illness: The dynamo of volition in health care. Current directions in psychological science. 2012; 21:60-65.

18. Broadbent E, Petrie KJ, Ellis CJ, Ying J, Gamble G. A picture of health-myocardial infarction patients' drawings of their hearts and subsequent disability: A longitudinal study. J Psychosom Res. 2004; 57:583-587.

19. Broadbent E, Ellis CJ, Gamble G, Petrie KJ. Changes in patient drawings of the heart identify slow recovery after myocardial infarction. Psychosom Med. 2006; 68:910-913.

20. Saint Arnault D, Shimabukuro S. The clinical ethnographic interview: A user-friendly guide to the cultural formulation of distress and help seeking. Transcult Psychiatry. 2012; 49:302-322.

21. Brett-Maclean P. Body mapping: Embodying the self living with HIV/ AIDS. CMAJ. 2009; 180:740-741.

22. Biktimirov EN, Nilson LB. Show them the money: Using mind mapping in the introductory finance course. Journal of Financial Education. 2006; :72-86.

23. Buzan T, Buzan B. The mind map book: How to use radiant thinking to maximize your brain \'s untapped potential. 1996; .

24. Kleinman A. The Illness Narratives: Suffering, Healing, and the Human Condition. Basic books, 1988.

25. Aggarwal NK, Nicasio AV, DeSilva R, Boiler M, Lewis-Fernández R. Barriers to implementing the DSM-5 cultural formulation interview: A qualitative study. Culture, Medicine, and Psychiatry. 2013; 37:505-533.

26. Cohen MZ, Tripp-Reimer T, Smith C, Sorofman B, Lively S. Explanatory models of diabetes: Patient practitioner variation. Soc Sci Med. 1994; 38:59-66.

27. Braun V, Clarke v. Using thematic analysis in psychology. Qualitative research in psychology. 2006; 3:77-101.

28. Pennebaker JW, Beall SK. Confronting a traumatic event: Toward an understanding of inhibition and disease. J Abnorm Psychol. 1986; 95:274

29. Horowitz S. Evidence-based health outcomes of expressive writing. Alternative and Complementary Therapies. 2008; 14:194-198.

30. Sakalys JA. Restoring the patient's voice. the therapeutics of illness narratives. J Holist Nurs. 2003; 21:228-241.

31. Abi-El-Mona I, Adb-El-Khalick F. The influence of mind mapping on eighth graders' science achievement. School Science and mathematics. 2008; 108:298-312.

32. Dubbin LA, Chang JS, Shim JK. Cultural health capital and the interactional dynamics of patient-centered care. Soc Sci Med. 2013; 93:113-120.

33. Pennebaker JW, Seagal JD. Forming a story: The health benefits of narrative. J Clin Psychol. 1999; 55:1243-1254.

34. DeSalvo LA. Writing as a Way of Healing: How Telling our Stories Transforms our Lives. Beacon Press, 1999. 